\title{
Disulfide Chromophores Arise from Stereoelectronic Effects
}

Henry R. Kilgore and Ronald T. Raines*

Department of Chemistry, Massachusetts Institute of Technology, Cambridge, Massachusetts 02139, United States

*rtraines@mit.edu

\begin{tabular}{ll}
\hline \multicolumn{1}{c}{ Content } & Page \\
\hline Table of Contents & $\mathrm{S} 1$ \\
Figure S1. UV-vis spectroscopy of oxidized lipoic acid and oxidized dithiothreitol & $\mathrm{S} 2$ \\
Figure S2. Dependence of absorbance on conformation and environment & $\mathrm{S} 3$ \\
Table S1. Physical and Photophysical Properties of Disulfides & $\mathrm{S} 4$ \\
Tables S2-S24. Atomic Coordinates of Disulfides & $\mathrm{S} 5$ \\
Tables S25-S28. Anomeric Conformations of $\mathrm{XH}_{2} \mathrm{C}-\mathrm{S}-\mathrm{S}-\mathrm{CH}_{2} \mathrm{X}$ & $\mathrm{S} 15$ \\
Tables S29-S32. Agnostic Conformations of $\mathrm{XH}_{2} \mathrm{C}-\mathrm{S}-\mathrm{S}-\mathrm{CH}_{2} \mathrm{X}$ & $\mathrm{S} 16$ \\
\hline
\end{tabular}



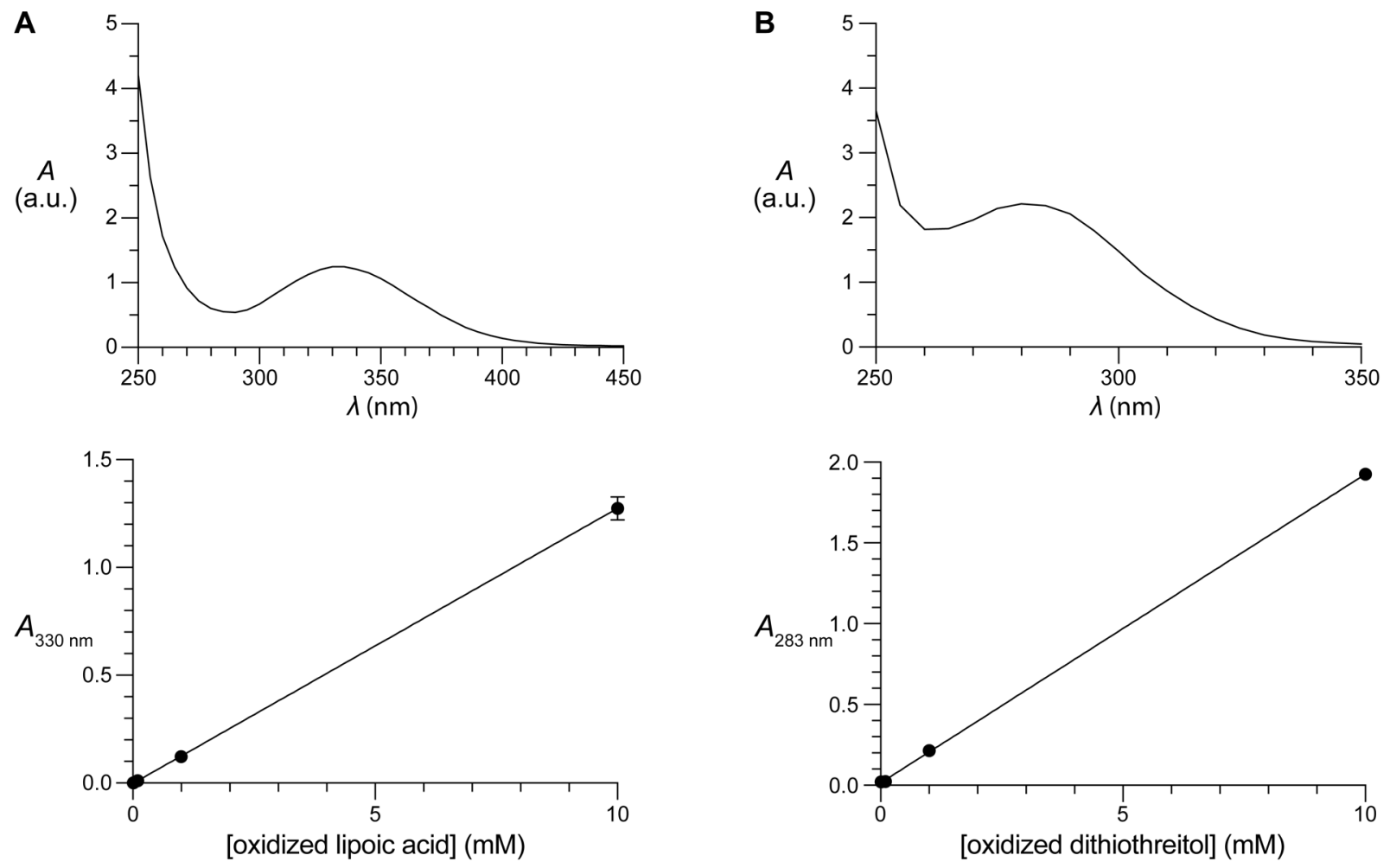

Figure S1. UV-vis spectroscopy of oxidized lipoic acid and oxidized dithiothreitol. (A) Spectrum of $10 \mathrm{mM}$ oxidized lipoic acid $\left(\lambda_{\max }=330 \mathrm{~nm}\right)$ and concentration-dependence of absorbance at $330 \mathrm{~nm}\left(\varepsilon=128 \mathrm{M}^{-1} \mathrm{~cm}^{-1}\right)$. (B) Spectrum of $10 \mathrm{mM}$ oxidized dithiothreitol $\left(\lambda_{\max }=283 \mathrm{~nm}\right)$ and concentration-dependence of absorbance at $283 \mathrm{~nm}\left(\varepsilon=191 \mathrm{M}^{-1} \mathrm{~cm}^{-1}\right)$. Data were obtained in $20 \mathrm{mM}$ BIS-TRIS buffer, $\mathrm{pH}$ 7.5. 


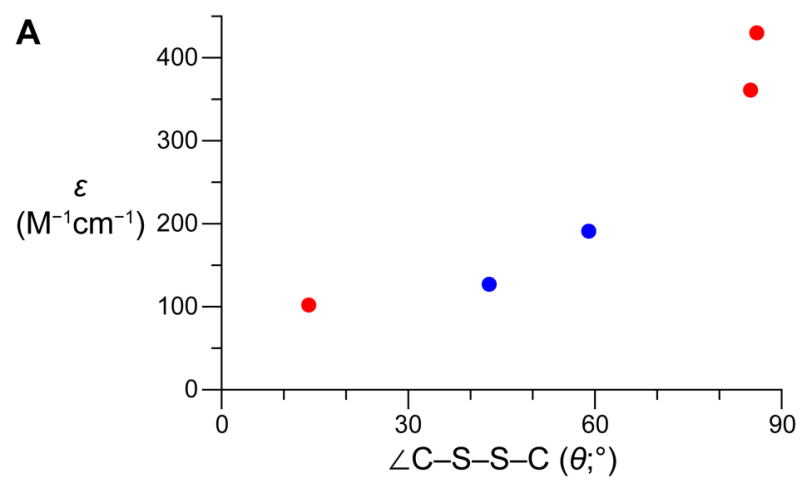

Figure S2. Dependence of the absorbance of

B

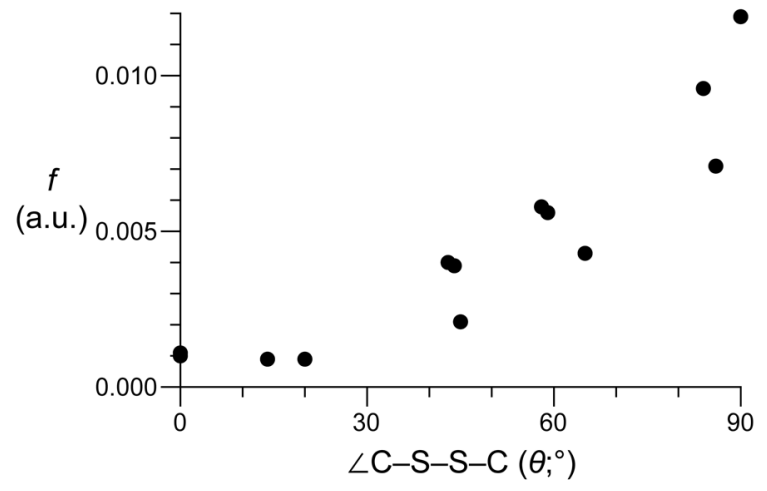

C

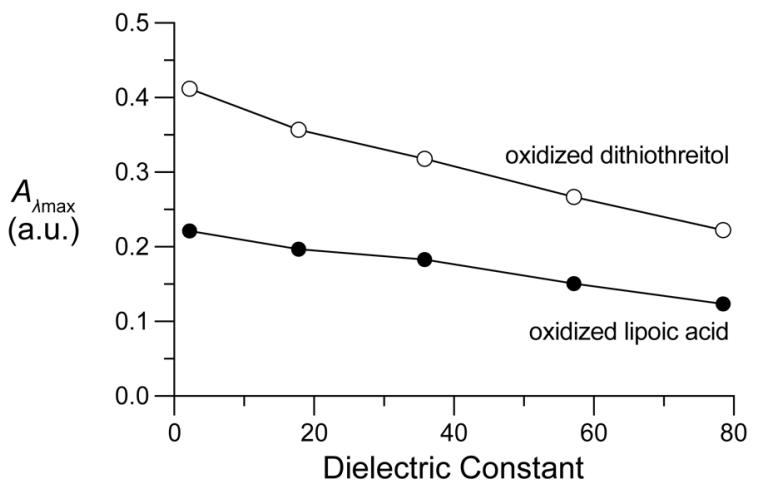

disulfide bonds on their conformation and environment. (A) Graph of experimental extinction coefficients at $\lambda_{\max }$ versus the computed $\mathrm{C}-\mathrm{S}-\mathrm{S}-\mathrm{C}$ dihedral angle. Values were recorded in water (blue; Figure $\mathrm{S} 1$ ) or hexane (red). (B) Graph of computed oscillator strengths versus the computed $\mathrm{C}-\mathrm{S}-$ S-C dihedral angle. (C) Graph of experimental absorbances of oxidized lipoic acid and oxidized dithiothreitol at $\lambda_{\max }$ versus the dielectric constant of the solution. Values are listed in Table S1. 
Table S1. Physical and Photophysical Properties of Disulfides.

\begin{tabular}{|c|c|c|c|c|c|}
\hline Compound & $\begin{array}{c}\angle \mathrm{C}-\mathrm{S}-\mathrm{S}-\mathrm{C}^{\mathrm{comp}} \\
\left(\theta,^{\circ}\right)\end{array}$ & $\begin{array}{c}\lambda_{\max }^{\exp } \\
(\mathrm{nm})\end{array}$ & $\begin{array}{c}\lambda_{\max }{ }^{\text {comp }} \\
(\mathrm{nm})\end{array}$ & $\begin{array}{c}\varepsilon^{\exp } \\
\left(\mathrm{M}^{-1} \mathrm{~cm}^{-1}\right)\end{array}$ & $\begin{array}{c}f \\
\text { (a.u.) }\end{array}$ \\
\hline dithiirane & 0 & & 458 & & 0.0010 \\
\hline 1,2-dithietane & 20 & & 449 & & 0.0009 \\
\hline 1,2-dithiolane & 44 & & 324 & & 0.0039 \\
\hline 1,2-dithiane & 58 & & 304 & & 0.0058 \\
\hline 1,2-dithiepane & 84 & & 270 & & 0.0096 \\
\hline 1,2-dithiocane & 90 & & 266 & & 0.0119 \\
\hline 1 & 64 & & 286 & & 0.0040 \\
\hline 2 & 68 & & 281 & & 0.0010 \\
\hline 3 & 88 & & 268 & & 0.0013 \\
\hline 4 & 0 & $452^{1}$ & 503 & & 0.0011 \\
\hline 5 & 14 & $426^{2}$ & 473 & $102^{2}$ & 0.0009 \\
\hline oxidized lipoic acid & 43 & 330 & 320 & 128 & 0.0040 \\
\hline oxidized dithiothreitol & 59 & 283 & 302 & 191 & 0.0056 \\
\hline dimethyl disulfide & 85 & $250^{3}$ & 266 & $361^{4}$ & 0.0087 \\
\hline diethyl disulfide & 86 & $248^{5}$ & 266 & $430^{6}$ & 0.0071 \\
\hline diethyl disulfide $\left(\theta=45^{\circ}\right)$ & 45 & & 325 & & 0.0021 \\
\hline diethyl disulfide $\left(\theta=65^{\circ}\right)$ & 65 & & 291 & & 0.0043 \\
\hline $\mathrm{CF}_{3} \mathrm{SSCF}_{3}$ & 83 & & 258 & & 0.0070 \\
\hline
\end{tabular}

(1) Ishii, A.; Akazawa, T.; Maruta, T.; Nakayama, J.; Hoshino, M.; Shiro, M. The First Isolable Dithiirane by Oxidation of a Dithietane. Angew. Chem., Int. Ed. 1994, 33, 777-779.

(2) Nicolaou, K. C.; Hwang, C. K.; Duggan, M. E.; Carrol, P. J. Dithiatopazine. The First Stable 1,2-Dithietane. J. Am. Chem. Soc. 1987, 109, 3801-3802.

(3) Hearn, C. H.; Turcu, E.; Joens, J. A. The Near U.V. Absorption Spectra of Dimethyl Sulfide, Diethyl Sulfide, and Dimethyl Disulfide at T=300K. Atmos. Environ. 1990, 24A, 1939-1944.

(4) Rosenthal, N. A.; Oster, G. A. Ultraviolet Spectra of Alkyl Disulfides and Their Relation to Alkali Cleavage of Disulfide Bonds. J. Am. Chem. Soc. 1961, 83, 4445-4448.

(5) Keller-Rudek, H.; Moortgat, G. K.; Sander, R.; Sörensen, R. The MPI-Mainz US/VIS Spectral Atlas of Gaseous Molecules of Atmospheric Interest. Earth Syst. Sci. Data 2013, 5, 365373.

(6) Ley, H.; Arends, B. Absorptionsspektren von Schwefelverbindungen in verschiedenen Wertigkeitsstufen. Z. Phys. Chem. 1932, B15, 311-325. 
Table S2. Dithiirane $(n=1)$<smiles>C1SS1</smiles>

$\begin{array}{lrrr}\mathrm{C} & 0.00000000 & 0.00000000 & 1.13826100 \\ \mathrm{H} & 0.92046200 & 0.00000000 & 1.70660400 \\ \mathrm{H} & -0.92046200 & 0.00000000 & 1.70660400 \\ \mathrm{~S} & 0.00000000 & 1.04575800 & -0.32008700 \\ \mathrm{~S} & 0.00000000 & -1.04575800 & -0.32008700\end{array}$

Table S3. 1,2-Dithietane $(n=2)$<smiles>[S]S</smiles>

$\begin{array}{lrrr}\mathrm{C} & 0.00000000 & 0.00000000 & 1.13826100 \\ \mathrm{H} & 0.92046200 & 0.00000000 & 1.70660400 \\ \mathrm{H} & -0.92046200 & 0.00000000 & 1.70660400 \\ \mathrm{~S} & 0.00000000 & 1.04575800 & -0.32008700 \\ \mathrm{~S} & 0.00000000 & -1.04575800 & -0.32008700\end{array}$

Table S4. 1,2-Dithiolane $(n=3)$<smiles>C1CSSC1</smiles>

$\begin{array}{lrrr}\mathrm{C} & 1.33780400 & -0.71300900 & -0.27852800 \\ \mathrm{C} & 1.42657300 & 0.82704700 & -0.11497100 \\ \mathrm{C} & 0.13863100 & 1.40153900 & 0.48072000 \\ \mathrm{H} & 1.51854600 & -0.99871600 & -1.31355100 \\ \mathrm{H} & 2.04780400 & -1.24092800 & 0.35827400 \\ \mathrm{H} & 1.58643600 & 1.27366700 & -1.09705400 \\ \mathrm{H} & 2.27845000 & 1.09805400 & 0.51283500 \\ \mathrm{H} & 0.08750400 & 1.25271800 & 1.55992800 \\ \mathrm{H} & 0.02246100 & 2.46185200 & 0.25136100 \\ \mathrm{~S} & -0.31941200 & -1.30301700 & 0.23913300 \\ \mathrm{~S} & -1.24054100 & 0.49426100 & -0.28882800\end{array}$

Table S5. 1,2-Dithiane $(n=4)$

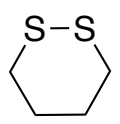

$\begin{array}{lrrr}\text { C } & -1.65511600 & 0.75161400 & 0.14610000 \\ \text { C } & -0.46020900 & 1.50607800 & -0.42836800 \\ \text { C } & -0.46025400 & -1.50606600 & 0.42837400 \\ \text { C } & -1.65513700 & -0.75157400 & -0.14610500 \\ \text { H } & -0.38424600 & 1.37726500 & -1.50916900 \\ \text { H } & -0.53787700 & 2.57299500 & -0.21101200 \\ \text { H } & -1.68641200 & 0.91396300 & 1.22728800 \\ \text { H } & -2.56835100 & 1.18662900 & -0.26994500 \\ \text { H } & -0.53794200 & -2.57298200 & 0.21103300\end{array}$




$\begin{array}{lrrr}\mathrm{H} & -0.38428800 & -1.37723600 & 1.50917300 \\ \mathrm{H} & -1.68643500 & -0.91392800 & -1.22729200 \\ \mathrm{H} & -2.56838700 & -1.18656000 & 0.26994100 \\ \mathrm{~S} & 1.11680700 & -0.98442900 & -0.31949200 \\ \mathrm{~S} & 1.11683300 & 0.98440100 & 0.31949100\end{array}$

Table S6. 1,2-Dithiepane $(n=5)$<smiles>C1CCSSCC1</smiles>

$\begin{array}{lrrr}\mathrm{C} & -1.33283100 & 1.30096600 & -0.38217600 \\ \mathrm{C} & -2.03033700 & -0.02936500 & -0.07159800 \\ \mathrm{C} & -1.23128200 & -1.29279500 & -0.35779200 \\ \mathrm{C} & -0.23309900 & 1.69828500 & 0.61818100 \\ \mathrm{C} & 1.19929400 & 1.47030100 & 0.15246400 \\ \mathrm{H} & -0.92541900 & 1.27744400 & -1.39775000 \\ \mathrm{H} & -2.33820500 & -0.02930800 & 0.97816400 \\ \mathrm{H} & -0.98657200 & -1.38010700 & -1.41667300 \\ \mathrm{H} & -0.38587900 & 1.16676800 & 1.55942400 \\ \mathrm{H} & 1.89892600 & 1.67119500 & 0.96301000 \\ \mathrm{H} & -2.10198500 & 2.07578000 & -0.37747400 \\ \mathrm{H} & -2.94865300 & -0.08949400 & -0.66495800 \\ \mathrm{H} & -1.80605400 & -2.17621800 & -0.06957400 \\ \mathrm{H} & -0.31585800 & 2.76305400 & 0.85352900 \\ \mathrm{H} & 1.44322100 & 2.13399900 & -0.68088300 \\ \mathrm{~S} & 1.54896000 & -0.19434100 & -0.52935000 \\ \mathrm{~S} & 0.34079100 & -1.44925000 & 0.56052000\end{array}$

Table S7. 1,2-Dithiocane $(n=6)$<smiles>C1CCCSSCC1</smiles>

$\begin{array}{lrrr}\mathrm{C} & -1.58989200 & -0.91662500 & 0.74757100 \\ \mathrm{C} & -0.25084300 & 1.97003900 & -0.14888200 \\ \mathrm{C} & -2.20667500 & 0.26131800 & -0.01943000 \\ \mathrm{C} & -1.26632200 & 1.10405500 & -0.88220000 \\ \mathrm{H} & -0.73312300 & 2.55520500 & 0.63996200 \\ \mathrm{H} & -0.96911800 & -0.55059700 & 1.56713900 \\ \mathrm{H} & -2.72125200 & 0.91558000 & 0.69327800 \\ \mathrm{H} & -2.41644600 & -1.46021900 & 1.21309500 \\ \mathrm{H} & 0.21282900 & 2.66544500 & -0.84918400 \\ \mathrm{H} & -2.98310100 & -0.13910400 & -0.68062900 \\ \mathrm{H} & -1.87840500 & 1.78843800 & -1.47934400 \\ \mathrm{H} & -0.73796200 & 0.47297900 & -1.60242300 \\ \mathrm{C} & 0.73673200 & -1.85535600 & 0.09392700 \\ \mathrm{H} & 0.97625300 & -1.94362300 & 1.15565300 \\ \mathrm{H} & 1.21389700 & -2.69288700 & -0.42278300 \\ \mathrm{C} & -0.77517600 & -1.89810100 & -0.12100400\end{array}$




$\begin{array}{lrrr}H & -0.99803800 & -1.75079300 & -1.18259000 \\ H & -1.08540200 & -2.92164300 & 0.11208400 \\ \mathrm{~S} & 1.65282600 & -0.41428600 & -0.55906000 \\ \mathrm{~S} & 1.11173200 & 1.10611400 & 0.73505100\end{array}$

Table S8. Disulfide 1<smiles>FC(F)(F)C(F)(F)SC(F)(F)C(F)(F)C(F)(F)C(F)(F)C(F)(F)F</smiles>

$\begin{array}{lrrr}\text { C } & 2.18500400 & 0.01637000 & -0.11438900 \\ \text { C } & -2.18004300 & -0.02904600 & -0.10326500 \\ \text { S } & -0.72037400 & -0.78540000 & -0.97485400 \\ \text { S } & 0.75837000 & 0.66115000 & -1.10339100 \\ \text { C } & 3.24616400 & 1.15929400 & -0.18718000 \\ \text { C } & -3.39993300 & -0.48306700 & -0.96196200 \\ \text { C } & 2.76844100 & -1.25636100 & -0.79316000 \\ \text { C } & 1.86930900 & -0.26823800 & 1.37565700 \\ \text { C } & -2.17234100 & 1.52007200 & -0.04137900 \\ \text { C } & -2.32238600 & -0.62252600 & 1.32473800 \\ \text { F } & -3.48887500 & 0.25542500 & -2.06220000 \\ \text { F } & -3.26418500 & -1.75762100 & -1.32784900 \\ \text { F } & -4.53627300 & -0.36976000 & -0.28438800 \\ \text { F } & -1.19745500 & -0.50004800 & 2.01012600 \\ \text { F } & -2.62227600 & -1.91751100 & 1.24912200 \\ \text { F } & -3.28717600 & -0.00978600 & 2.00768100 \\ \text { F } & -3.39048300 & 1.97362600 & 0.23894400 \\ \text { F } & -1.80405700 & 2.05249700 & -1.20341300 \\ \text { F } & -1.34101100 & 1.95212900 & 0.90120000 \\ \text { F } & 1.30701600 & -1.46797400 & 1.50576300 \\ \text { F } & 1.05209600 & 0.64631200 & 1.87465200 \\ \text { F } & 2.98571200 & -0.25759400 & 2.10262300 \\ \text { F } & 1.81416600 & -2.14392200 & -1.06735800 \\ \text { F } & 3.36059800 & -0.93191400 & -1.93916000 \\ \text { F } & 3.66119600 & -1.85243400 & -0.00955400 \\ \text { F } & 4.47153300 & 0.69303800 & 0.02923000 \\ \text { F } & 3.23467100 & 1.74122900 & -1.38543400 \\ \text { F } & 2.98111000 & 2.09086300 & 0.72196500\end{array}$

Table S9. Disulfide 2<smiles>CC(C)(C)SSC(C)(C)C</smiles>

$\begin{array}{lrrr}\text { C } & -2.12237100 & 0.18281400 & -0.06051800 \\ \text { C } & 2.12236600 & 0.18283800 & 0.06049100 \\ \text { S } & 0.75430200 & -0.81570400 & -0.71040500 \\ \text { S } & -0.75427100 & -0.81557200 & 0.71047900 \\ \text { C } & -3.23476200 & 0.11896400 & 0.98811100 \\ \text { H } & -2.92241700 & 0.58274500 & 1.92663400\end{array}$




$\begin{array}{rrrr}\mathrm{H} & -4.10966500 & 0.65822900 & 0.61429000 \\ \mathrm{H} & -3.53049700 & -0.91314100 & 1.18948200 \\ \mathrm{C} & 3.23478200 & 0.11894800 & -0.98810200 \\ \mathrm{H} & 4.10966900 & 0.65822600 & -0.61427000 \\ \mathrm{H} & 3.53055500 & -0.91316600 & -1.18939700 \\ \mathrm{H} & 2.92245800 & 0.58267500 & -1.92665500 \\ \mathrm{C} & -2.57170100 & -0.48058800 & -1.35961000 \\ \mathrm{H} & -3.41966600 & 0.07098900 & -1.77791100 \\ \mathrm{H} & -1.76659500 & -0.47569600 & -2.09747200 \\ \mathrm{H} & -2.87870600 & -1.51343000 & -1.18516600 \\ \mathrm{C} & -1.70486600 & 1.62481200 & -0.31503600 \\ \mathrm{H} & -1.41451600 & 2.12376000 & 0.61146100 \\ \mathrm{H} & -0.87024700 & 1.66762600 & -1.01745700 \\ \mathrm{H} & -2.54532700 & 2.17230500 & -0.75327900 \\ \mathrm{C} & 2.57172600 & -0.48043900 & 1.35964300 \\ \mathrm{H} & 2.87887600 & -1.51325300 & 1.18528100 \\ \mathrm{H} & 3.41961900 & 0.07125100 & 1.77794700 \\ \mathrm{H} & 1.76661500 & -0.47560300 & 2.09750300 \\ \mathrm{C} & 1.70476600 & 1.62483800 & 0.31487500 \\ \mathrm{H} & 1.41444000 & 2.12369300 & -0.61168000 \\ \mathrm{H} & 0.87011200 & 1.66766300 & 1.01725400 \\ \mathrm{H} & 2.54516500 & 2.17242800 & 0.75311500\end{array}$

Table S10. Disulfide 3<smiles>COC(OC)(OC)SSC(OC)(OC)OC</smiles>

$\begin{array}{lrrr}\mathrm{C} & -2.13327800 & -0.04536700 & 0.29982400 \\ \mathrm{C} & 1.91300100 & -0.06907900 & -0.08284900 \\ \mathrm{~S} & 0.65355100 & 1.23332800 & -0.62859100 \\ \mathrm{~S} & -0.96680400 & 0.12733400 & -1.23997100 \\ \mathrm{O} & -2.99595800 & -1.05529300 & -0.05260300 \\ \mathrm{O} & -1.42527300 & -0.38089700 & 1.40980400 \\ \mathrm{O} & -2.85278100 & 1.09334700 & 0.52857200 \\ \mathrm{O} & 1.75520300 & -1.26314300 & -0.72600300 \\ \mathrm{O} & 1.71031600 & -0.25546600 & 1.26584900 \\ \mathrm{O} & 3.18486300 & 0.41544200 & -0.36918900 \\ \mathrm{C} & -0.89394100 & -1.70302400 & 1.44401300 \\ \mathrm{H} & -0.28711800 & -1.89720200 & 0.55653300 \\ \mathrm{H} & -0.26731500 & -1.74020700 & 2.33163200 \\ \mathrm{H} & -1.70411500 & -2.42904200 & 1.51596500 \\ \mathrm{C} & -4.03397100 & -0.71820600 & -0.96898000 \\ \mathrm{H} & -3.62844600 & -0.24354500 & -1.86588400 \\ \mathrm{H} & -4.50568400 & -1.66136800 & -1.23550000 \\ \mathrm{H} & -4.75696700 & -0.05129700 & -0.50063700 \\ \mathrm{C} & -2.13732500 & 2.26131100 & 0.91000900 \\ \mathrm{H} & -1.35324700 & 2.02045800 & 1.62835700 \\ \mathrm{H} & -1.70363200 & 2.74110600 & 0.02979800\end{array}$




$\begin{array}{lrrr}\mathrm{H} & -2.87282000 & 2.92379400 & 1.36194600 \\ \mathrm{C} & 2.53443100 & -1.26261300 & 1.85267800 \\ \mathrm{H} & 3.58768500 & -1.09064100 & 1.61835000 \\ \mathrm{H} & 2.37713900 & -1.18315800 & 2.92672400 \\ \mathrm{H} & 2.23989700 & -2.25293300 & 1.50182900 \\ \mathrm{C} & 3.54728100 & 1.63410600 & 0.26576100 \\ \mathrm{H} & 3.35651800 & 1.58677600 & 1.34002900 \\ \mathrm{H} & 4.61196900 & 1.76327200 & 0.08276600 \\ \mathrm{H} & 3.00376400 & 2.47813000 & -0.16744500 \\ \mathrm{C} & 2.09987100 & -1.29789400 & -2.10741200 \\ \mathrm{H} & 3.18060800 & -1.23071300 & -2.23241100 \\ \mathrm{H} & 1.73072700 & -2.25116400 & -2.47882100 \\ \mathrm{H} & 1.61571600 & -0.48019700 & -2.64596000\end{array}$

Table S11. Disulfide 4<smiles>CC(C)(C)C1(c2ccccc2)SS1</smiles>

$\begin{array}{lrrr}\text { C } & 0.60938000 & -0.22770400 & -0.00013900 \\ \text { S } & 1.26763100 & -1.55513600 & -1.04960200 \\ \text { S } & 1.26774700 & -1.55528500 & 1.04904000 \\ \text { C } & -0.89827500 & -0.13238200 & -0.00004800 \\ \text { C } & -1.60465900 & -0.06947500 & -1.20060600 \\ \text { C } & -1.60466600 & -0.07024800 & 1.20054700 \\ \text { C } & -2.98811000 & 0.06677800 & -1.20225300 \\ \text { H } & -1.06707800 & -0.14027300 & -2.13990700 \\ \mathrm{C} & -2.98812300 & 0.06599600 & 1.20225400 \\ \text { H } & -1.06708700 & -0.14175200 & 2.13979600 \\ \mathrm{C} & -3.68373400 & 0.13770300 & 0.00002200 \\ \mathrm{H} & -3.52246500 & 0.10792600 & -2.14385800 \\ \mathrm{H} & -3.52249900 & 0.10652600 & 2.14387400 \\ \mathrm{H} & -4.76252600 & 0.23755400 & 0.00005100 \\ \mathrm{C} & 1.37016000 & 1.12610100 & 0.00018200 \\ \mathrm{C} & 2.89254200 & 0.95190300 & -0.00226900 \\ \mathrm{H} & 3.23390800 & 0.41725100 & -0.88917000 \\ \mathrm{H} & 3.35556500 & 1.94130800 & 0.00033200 \\ \mathrm{H} & 3.23619700 & 0.41149900 & 0.88026600 \\ \mathrm{C} & 0.96785300 & 1.91821200 & -1.25202500 \\ \mathrm{H} & -0.09744200 & 2.15478800 & -1.26125800 \\ \mathrm{H} & 1.52353200 & 2.85862100 & -1.27878500 \\ \mathrm{H} & 1.21141800 & 1.35853000 & -2.16035000 \\ \mathrm{C} & 0.97131300 & 1.91535800 & 1.25529800 \\ \mathrm{H} & 1.52609700 & 2.85629300 & 1.28209300 \\ \mathrm{H} & -0.09416600 & 2.15094000 & 1.26845900 \\ \mathrm{H} & 1.21842500 & 1.35407900 & 2.16166700\end{array}$


Table S12. Disulfide 5<smiles>C1CCC23OCCCCC2(CCCS3)OC1</smiles>

$\begin{array}{lrrr}\mathrm{C} & 0.60938000 & -0.22770400 & -0.00013900 \\ \mathrm{~S} & 1.26763100 & -1.55513600 & -1.04960200 \\ \mathrm{~S} & 1.26774700 & -1.55528500 & 1.04904000 \\ \mathrm{C} & -0.89827500 & -0.13238200 & -0.00004800 \\ \mathrm{C} & -1.60465900 & -0.06947500 & -1.20060600 \\ \mathrm{C} & -1.60466600 & -0.07024800 & 1.20054700 \\ \mathrm{C} & -2.98811000 & 0.06677800 & -1.20225300 \\ \mathrm{H} & -1.06707800 & -0.14027300 & -2.13990700 \\ \mathrm{C} & -2.98812300 & 0.06599600 & 1.20225400 \\ \mathrm{H} & -1.06708700 & -0.14175200 & 2.13979600 \\ \mathrm{C} & -3.68373400 & 0.13770300 & 0.00002200 \\ \mathrm{H} & -3.52246500 & 0.10792600 & -2.14385800 \\ \mathrm{H} & -3.52249900 & 0.10652600 & 2.14387400 \\ \mathrm{H} & -4.76252600 & 0.23755400 & 0.00005100 \\ \mathrm{C} & 1.37016000 & 1.12610100 & 0.00018200 \\ \mathrm{C} & 2.89254200 & 0.95190300 & -0.00226900 \\ \mathrm{H} & 3.23390800 & 0.41725100 & -0.88917000 \\ \mathrm{H} & 3.35556500 & 1.94130800 & 0.00033200 \\ \mathrm{H} & 3.23619700 & 0.41149900 & 0.88026600 \\ \mathrm{C} & 0.96785300 & 1.91821200 & -1.25202500 \\ \mathrm{H} & -0.09744200 & 2.15478800 & -1.26125800 \\ \mathrm{H} & 1.52353200 & 2.85862100 & -1.27878500 \\ \mathrm{H} & 1.21141800 & 1.35853000 & -2.16035000 \\ \mathrm{C} & 0.97131300 & 1.91535800 & 1.25529800 \\ \mathrm{H} & 1.52609700 & 2.85629300 & 1.28209300 \\ \mathrm{H} & -0.09416600 & 2.15094000 & 1.26845900 \\ \mathrm{H} & 1.21842500 & 1.35407900 & 2.16166700\end{array}$

Table S13. Oxidized Lipoic Acid

$\begin{array}{llll}\mathrm{C} & -2.78908473 & 6.56376113 & -6.93561926 \\ \mathrm{C} & -2.11901871 & 7.09641643 & -5.64190632 \\ \mathrm{C} & -2.75591125 & 6.48693542 & -4.39027370 \\ \mathrm{H} & -3.16869619 & 7.38824550 & -7.53702546 \\ \mathrm{H} & -2.10564798 & 5.97274683 & -7.54548117 \\ \mathrm{H} & -2.24346877 & 8.17930614 & -5.60482935 \\ \mathrm{H} & -1.04672552 & 6.88813208 & -5.65110092 \\ \mathrm{H} & -2.39742577 & 5.47405654 & -4.20314778 \\ \mathrm{~S} & -4.16761302 & 5.43319197 & -6.50675987 \\ \mathrm{~S} & -4.54873360 & 6.41171024 & -4.70268704 \\ \mathrm{C} & -2.51752289 & 7.35828602 & -3.14306918 \\ \mathrm{H} & -1.46616506 & 7.44453259 & -2.96388148 \\ \mathrm{H} & -2.93307234 & 8.33124198 & -3.30299215 \\ \mathrm{C} & -3.19422337 & 6.70517660 & -1.92359202 \\ \mathrm{H} & -4.21088485 & 7.03301218 & -1.86175948\end{array}$




$\begin{array}{llll}\mathrm{H} & -3.16851252 & 5.64069301 & -2.02901323 \\ \mathrm{C} & -2.44469554 & 7.11228977 & -0.64138030 \\ \mathrm{H} & -2.39208056 & 8.17937610 & -0.58257184 \\ \mathrm{H} & -1.45464875 & 6.70702835 & -0.66307204 \\ \mathrm{C} & -3.19582136 & 6.56686970 & 0.58741055 \\ \mathrm{H} & -4.18291275 & 6.97899347 & 0.61395447 \\ \mathrm{H} & -3.25614438 & 5.50044415 & 0.52418326 \\ \mathrm{C} & -2.43945322 & 6.96315514 & 1.86899749 \\ \mathrm{O} & -1.16349295 & 7.60184839 & 1.77467710 \\ \mathrm{H} & -0.80134619 & 7.72585644 & 2.65505868 \\ \mathrm{O} & -2.94038355 & 6.72685600 & 2.99895416\end{array}$

Table S14. Oxidized Dithiothreitol

$\begin{array}{rrrr}\mathrm{C} & -1.07073500 & 0.71401800 & 0.21060400 \\ \mathrm{C} & 0.11083300 & 1.52467700 & -0.31731400 \\ \mathrm{C} & 0.14790700 & -1.53058700 & 0.30743500 \\ \mathrm{C} & -1.05546700 & -0.75516100 & -0.21260100 \\ \mathrm{H} & 0.16455500 & 1.46214900 & -1.40580600 \\ \mathrm{H} & -0.00003900 & 2.57426000 & -0.03038400 \\ \mathrm{H} & -1.08480300 & 0.75143200 & 1.30731900 \\ \mathrm{H} & 0.04801500 & -2.57555700 & 0.00709300 \\ \mathrm{H} & 0.19728700 & -1.48096200 & 1.39654800 \\ \mathrm{H} & -1.05800400 & -0.78642000 & -1.31121300 \\ \mathrm{~S} & 1.70950200 & -0.93872100 & -0.40528200 \\ \mathrm{~S} & 1.68493900 & 0.97595800 & 0.40809600 \\ \mathrm{O} & -2.19735000 & -1.40526200 & 0.30350200 \\ \mathrm{H} & -2.95823200 & -0.85743500 & 0.08100300 \\ \mathrm{O} & -2.29253900 & 1.22188600 & -0.31737200 \\ \mathrm{H} & -2.49596200 & 2.06605500 & 0.09264300\end{array}$

Table S15. $\mathrm{CF}_{3} \mathrm{SSCF}_{3}$

$\begin{array}{lrrr}\text { C } & -1.89556800 & 0.22704500 & -0.04673700 \\ \text { C } & 1.89554100 & 0.22708500 & 0.04663300 \\ \text { S } & 0.74088900 & -0.97131500 & -0.71372600 \\ \text { S } & -0.74081700 & -0.97096000 & 0.71408900 \\ \text { F } & 2.31288200 & -0.16794200 & 1.24500500 \\ \text { F } & 2.94905100 & 0.30551800 & -0.76323800 \\ \text { F } & 1.37367900 & 1.43769900 & 0.18344500 \\ \text { F } & -1.37359000 & 1.43750100 & -0.18431400 \\ \text { F } & -2.94884800 & 0.30611700 & 0.76331100 \\ \text { F } & -2.31328300 & -0.16871400 & -1.24478600\end{array}$

Table S16. Dimethyl Disulfide

$\begin{array}{lrrr}C & 1.78975000 & 0.81488600 & 0.39401600 \\ \text { C } & -1.78955100 & 0.81515300 & -0.39391400 \\ \text { S } & -0.91041000 & -0.50682300 & 0.49155900 \\ \text { S } & 0.91030200 & -0.50668600 & -0.49165900\end{array}$




$\begin{array}{rrrr}\mathrm{H} & 1.28778200 & 1.77252200 & 0.26321300 \\ \mathrm{H} & 1.86823600 & 0.57311500 & 1.45215000 \\ \mathrm{H} & 2.78740600 & 0.87186000 & -0.04329300 \\ \mathrm{H} & -2.78731400 & 0.87152600 & 0.04325700 \\ \mathrm{H} & -1.28786200 & 1.77282300 & -0.26211900 \\ \mathrm{H} & -1.86770400 & 0.57406600 & -1.45221100\end{array}$

Table S17. Diethyl Disulfide (unconstrained)

$\begin{array}{lrrr}\mathrm{C} & 0.46452400 & 1.79964100 & 0.57555300 \\ \mathrm{H} & 0.35524900 & 1.24779700 & 1.51001800 \\ \mathrm{H} & 1.51760800 & 1.80805700 & 0.29509200 \\ \mathrm{C} & -0.46452400 & -1.79964100 & 0.57555300 \\ \mathrm{H} & -1.51760800 & -1.80805700 & 0.29509200 \\ \mathrm{H} & -0.35524900 & -1.24779700 & 1.51001800 \\ \mathrm{~S} & 0.46452400 & -0.92280700 & -0.73375900 \\ \mathrm{~S} & -0.46452400 & 0.92280700 & -0.73375900 \\ \mathrm{C} & -0.08766700 & 3.21295800 & 0.70779000 \\ \mathrm{H} & -1.14402200 & 3.19930200 & 0.98254000 \\ \mathrm{H} & 0.45794500 & 3.75582000 & 1.48183100 \\ \mathrm{H} & 0.01536000 & 3.76304500 & -0.22939300 \\ \mathrm{C} & 0.08766700 & -3.21295800 & 0.70779000 \\ \mathrm{H} & -0.45794500 & -3.75582000 & 1.48183100 \\ \mathrm{H} & -0.01536000 & -3.76304500 & -0.22939300 \\ \mathrm{H} & 1.14402200 & -3.19930200 & 0.98254000\end{array}$

Table S18. Diethyl Disulfide $\left(\theta=0^{\circ}\right)$

$\begin{array}{lrrr}\mathrm{C} & 1.55067100 & 0.80883400 & -0.02819700 \\ \mathrm{H} & 1.18900700 & 1.28858100 & -0.93736100 \\ \mathrm{H} & 1.12823900 & 1.31261500 & 0.84058500 \\ \mathrm{C} & -1.55066300 & 0.80883400 & 0.02828100 \\ \mathrm{H} & -1.12810800 & 1.31265100 & -0.84043400 \\ \mathrm{H} & -1.18913900 & 1.28852600 & 0.93751500 \\ \mathrm{~S} & -1.06943200 & -0.94947600 & 0.01947600 \\ \mathrm{~S} & 1.06942300 & -0.94947700 & -0.01947500 \\ \mathrm{C} & 3.07492100 & 0.85364400 & 0.02377500 \\ \mathrm{H} & 3.51189300 & 0.34128600 & -0.83493400 \\ \mathrm{H} & 3.41239000 & 1.89171000 & 0.00992100 \\ \mathrm{H} & 3.44932700 & 0.38252900 & 0.93376900 \\ \mathrm{C} & -3.07491100 & 0.85365100 & -0.02385300 \\ \mathrm{H} & -3.41237100 & 1.89172000 & -0.00997300 \\ \mathrm{H} & -3.44922500 & 0.38260000 & -0.93391800 \\ \mathrm{H} & -3.51198800 & 0.34124700 & 0.83477500\end{array}$

Table S19. Diethyl Disulfide $\left(\theta=10^{\circ}\right)$

$\begin{array}{lrrr}\mathrm{C} & -1.54626900 & 0.80232300 & -0.10136900 \\ \mathrm{H} & -1.07713500 & 1.37566200 & 0.69702100 \\ \mathrm{H} & -1.22919900 & 1.19890100 & -1.06657800\end{array}$




$\begin{array}{lrrr}\mathrm{C} & 1.54626900 & 0.80232300 & 0.10136900 \\ \mathrm{H} & 1.22919800 & 1.19890100 & 1.06657800 \\ \mathrm{H} & 1.07713500 & 1.37566200 & -0.69702100 \\ \mathrm{~S} & 1.06720000 & -0.95094500 & -0.03596400 \\ \mathrm{~S} & -1.06720000 & -0.95094500 & 0.03596400 \\ \mathrm{C} & -3.06555100 & 0.86232100 & 0.02423900 \\ \mathrm{H} & -3.39391500 & 0.46924800 & 0.98758500 \\ \mathrm{H} & -3.40014200 & 1.89825100 & -0.05655800 \\ \mathrm{H} & -3.54800600 & 0.28518600 & -0.76625900 \\ \mathrm{C} & 3.06555100 & 0.86232200 & -0.02423900 \\ \mathrm{H} & 3.40014200 & 1.89825200 & 0.05655800 \\ \mathrm{H} & 3.54800600 & 0.28518600 & 0.76625900 \\ \mathrm{H} & 3.39391500 & 0.46924800 & -0.98758500\end{array}$

Table S20. Diethyl Disulfide $\left(\theta=25^{\circ}\right)$

$\begin{array}{lrrr}\mathrm{C} & 1.54624900 & 0.78522500 & 0.16640300 \\ \mathrm{H} & 1.05808000 & 1.44034600 & -0.55370800 \\ \mathrm{H} & 1.24787300 & 1.07317000 & 1.17620500 \\ \mathrm{C} & -1.54625100 & 0.78522200 & -0.16641400 \\ \mathrm{H} & -1.24788900 & 1.07315600 & -1.17622400 \\ \mathrm{H} & -1.05807000 & 1.44035000 & 0.55368300 \\ \mathrm{~S} & -1.04981100 & -0.94000900 & 0.14931000 \\ \mathrm{~S} & 1.04981100 & -0.94000800 & -0.14930900 \\ \mathrm{C} & 3.06175000 & 0.85989900 & 0.01137500 \\ \mathrm{H} & 3.36777700 & 0.55298000 & -0.99023500 \\ \mathrm{H} & 3.39859400 & 1.88576200 & 0.17165000 \\ \mathrm{H} & 3.56276900 & 0.21713800 & 0.73675300 \\ \mathrm{C} & -3.06174900 & 0.85990100 & -0.01136500 \\ \mathrm{H} & -3.39859400 & 1.88576200 & -0.17164600 \\ \mathrm{H} & -3.56278000 & 0.21713200 & -0.73672800 \\ \mathrm{H} & -3.36776200 & 0.55299300 & 0.99025300\end{array}$

Table S21. Diethyl Disulfide $\left(\theta=45^{\circ}\right)$

$\begin{array}{lrrr}\mathrm{C} & 1.58087000 & 0.75048200 & 0.24331700 \\ \mathrm{H} & 1.08793300 & 1.51281100 & -0.35926800 \\ \mathrm{H} & 1.30996800 & 0.89236300 & 1.29159700 \\ \mathrm{C} & -1.58089000 & 0.75044200 & -0.24340200 \\ \mathrm{H} & -1.31010100 & 0.89221800 & -1.29172600 \\ \mathrm{H} & -1.08787200 & 1.51281800 & 0.35905700 \\ \mathrm{~S} & -1.00414500 & -0.89399700 & 0.29655800 \\ \mathrm{~S} & 1.00415400 & -0.89399700 & -0.29655400 \\ \mathrm{C} & 3.09170700 & 0.81437400 & 0.05078100 \\ \mathrm{H} & 3.36176500 & 0.63198800 & -0.99119600 \\ \mathrm{H} & 3.45972000 & 1.80432500 & 0.32674700 \\ \mathrm{H} & 3.59745900 & 0.07346300 & 0.67152800 \\ \mathrm{C} & -3.09170500 & 0.81438300 & -0.05070600 \\ \mathrm{H} & -3.45972800 & 1.80431700 & -0.32671800 \\ \mathrm{H} & -3.59753900 & 0.07342700 & -0.67133300\end{array}$




\section{$\begin{array}{llll}H & -3.36165300 & 0.63209400 & 0.99131600\end{array}$}

Table S22. Diethyl Disulfide $\left(\theta=65^{\circ}\right)$

$\begin{array}{lrrr}\mathrm{C} & 1.67430700 & 0.68533100 & 0.36916800 \\ \mathrm{H} & 1.14928900 & 1.55579500 & -0.02448600 \\ \mathrm{H} & 1.52001900 & 0.63591700 & 1.44790000 \\ \mathrm{C} & -1.67430200 & 0.68534300 & -0.36915300 \\ \mathrm{H} & -1.51999300 & 0.63595100 & -1.44788300 \\ \mathrm{H} & -1.14929500 & 1.55580000 & 0.02452900 \\ \mathrm{~S} & -0.96412100 & -0.82106100 & 0.38179300 \\ \mathrm{~S} & 0.96411900 & -0.82106100 & -0.38179500 \\ \mathrm{C} & 3.15609400 & 0.75187700 & 0.01978000 \\ \mathrm{H} & 3.30428500 & 0.77408400 & -1.06161100 \\ \mathrm{H} & 3.59771300 & 1.65724100 & 0.44027600 \\ \mathrm{H} & 3.69218300 & -0.10934700 & 0.42183600 \\ \mathrm{C} & -3.15609600 & 0.75187300 & -0.01979100 \\ \mathrm{H} & -3.59771100 & 1.65724400 & -0.44027700 \\ \mathrm{H} & -3.69217300 & -0.10934400 & -0.42187500 \\ \mathrm{H} & -3.30430700 & 0.77405700 & 1.06159800\end{array}$

Table S23. Diethyl Disulfide $\left(\theta=80^{\circ}\right)$

$\begin{array}{lrrr}\mathrm{C} & 1.75145300 & 0.60452900 & 0.47259600 \\ \mathrm{H} & 1.18974400 & 1.52139600 & 0.29007900 \\ \mathrm{H} & 1.71764500 & 0.37894200 & 1.53847400 \\ \mathrm{C} & -1.75145400 & 0.60452700 & -0.47259700 \\ \mathrm{H} & -1.71764800 & 0.37893700 & -1.53847600 \\ \mathrm{H} & -1.18974400 & 1.52139400 & -0.29008600 \\ \mathrm{~S} & -0.94208200 & -0.76779000 & 0.42539700 \\ \mathrm{~S} & 0.94208200 & -0.76779000 & -0.42539600 \\ \mathrm{C} & 3.18538600 & 0.73816000 & -0.02387100 \\ \mathrm{H} & 3.21339900 & 0.95550400 & -1.09325100 \\ \mathrm{H} & 3.68776900 & 1.55239400 & 0.50165100 \\ \mathrm{H} & 3.74868000 & -0.17972500 & 0.15402900 \\ \mathrm{C} & -3.18538600 & 0.73816100 & 0.02387200 \\ \mathrm{H} & -3.68776800 & 1.55239400 & -0.50165100 \\ \mathrm{H} & -3.74868100 & -0.17972300 & -0.15402500 \\ \mathrm{H} & -3.21339600 & 0.95550700 & 1.09325200\end{array}$

Table S24. Diethyl Disulfide $\left(\theta=90^{\circ}\right)$

$\begin{array}{lrrr}\mathrm{C} & 1.82352400 & 0.55472600 & 0.50884600 \\ \mathrm{H} & 1.28947600 & 1.50271700 & 0.43246900 \\ \mathrm{H} & 1.82590000 & 0.23729700 & 1.55132200 \\ \mathrm{C} & -1.82352400 & 0.55472600 & -0.50884600 \\ \mathrm{H} & -1.82590000 & 0.23729700 & -1.55132200 \\ \mathrm{H} & -1.28947600 & 1.50271700 & -0.43247000 \\ \mathrm{~S} & -0.92246200 & -0.70450400 & 0.46567600 \\ \mathrm{~S} & 0.92246200 & -0.70450400 & -0.46567600\end{array}$




$\begin{array}{lrrr}\mathrm{C} & 3.23978700 & 0.67771800 & -0.03779000 \\ \mathrm{H} & 3.23299000 & 0.98932900 & -1.08394600 \\ \mathrm{H} & 3.79728800 & 1.42132900 & 0.53475500 \\ \mathrm{H} & 3.77100600 & -0.27327300 & 0.03284900 \\ \mathrm{C} & -3.23978700 & 0.67771800 & 0.03779000 \\ \mathrm{H} & -3.79728800 & 1.42132900 & -0.53475500 \\ \mathrm{H} & -3.77100600 & -0.27327300 & -0.03284900 \\ \mathrm{H} & -3.23298900 & 0.98932900 & 1.08394600\end{array}$

\section{Anomeric Conformations of $\mathrm{XH}_{2} \mathrm{C}-\mathrm{S}-\mathrm{S}-\mathrm{CH}_{2} \mathrm{X}$}

Table S25. $X=M e$

$\begin{array}{lrrr}\mathrm{C} & 1.63128800 & 0.80927500 & -0.31116800 \\ \mathrm{C} & -2.15524200 & -0.03157600 & 0.07345700 \\ \mathrm{~S} & -0.66914900 & -0.95204800 & 0.60787500 \\ \mathrm{~S} & 0.64641000 & -0.59354200 & -0.95145500 \\ \mathrm{H} & -2.50098600 & -0.46548700 & -0.86540400 \\ \mathrm{H} & 0.95431200 & 1.62859900 & -0.06784400 \\ \mathrm{H} & 2.23620200 & 1.11399500 & -1.16923200 \\ \mathrm{H} & -2.89116500 & -0.27098400 & 0.84537900 \\ \mathrm{C} & 2.50524900 & 0.44044800 & 0.87643400 \\ \mathrm{H} & 3.19985400 & -0.35879700 & 0.61378600 \\ \mathrm{H} & 3.08049000 & 1.30863900 & 1.20747500 \\ \mathrm{H} & 1.89043600 & 0.10185400 & 1.71227000 \\ \mathrm{C} & -1.95864600 & 1.46957900 & -0.05485400 \\ \mathrm{H} & -1.24104700 & 1.69373900 & -0.84641400 \\ \mathrm{H} & -2.90338100 & 1.95195200 & -0.31702100 \\ \mathrm{H} & -1.59677600 & 1.89958300 & 0.88108400\end{array}$

Table S26. $X=\mathrm{OMe}$

$\begin{array}{lrrr}\text { C } & -1.41349800 & 0.35767000 & 0.70100500 \\ \mathrm{C} & 2.03843100 & -0.36498600 & -0.34448400 \\ \mathrm{~S} & 0.55945000 & -1.27163300 & -0.84714200 \\ \mathrm{~S} & -0.52194800 & -1.20237100 & 0.91904400 \\ \mathrm{H} & 2.44541600 & -0.81272900 & 0.56894200 \\ \mathrm{H} & -0.69913000 & 1.11866500 & 0.37271700 \\ \mathrm{H} & -1.80095800 & 0.61370700 & 1.69701800 \\ \mathrm{H} & 2.75845000 & -0.48734500 & -1.16475800 \\ \mathrm{O} & 1.73086500 & 0.98682800 & -0.13544000 \\ \mathrm{O} & -2.45108400 & 0.19367000 & -0.22113000 \\ \mathrm{C} & 2.83334200 & 1.70464500 & 0.37698200 \\ \mathrm{H} & 3.68269200 & 1.67261800 & -0.31595600 \\ \mathrm{H} & 2.51417200 & 2.73774000 & 0.50227000 \\ \mathrm{H} & 3.14853000 & 1.30189400 & 1.34679700 \\ \mathrm{C} & -3.01823000 & 1.42879100 & -0.59813400 \\ \mathrm{H} & -3.81367800 & 1.21115900 & -1.30852100 \\ \mathrm{H} & -3.44261400 & 1.95351100 & 0.26709600 \\ \mathrm{H} & -2.27142100 & 2.07415200 & -1.07568800\end{array}$


Table S27. $\mathrm{X}=\mathrm{F}$

$\begin{array}{lrrr}\text { C } & -1.56625500 & 0.74592000 & 0.27004300 \\ \text { C } & 2.04396300 & 0.15683000 & 0.12206300 \\ \text { S } & 0.78569200 & -0.86629000 & -0.66086100 \\ \text { S } & -0.70152200 & -0.75513900 & 0.78218900 \\ \text { H } & 2.20246900 & -0.15366100 & 1.15369500 \\ \text { H } & -0.84895700 & 1.52319400 & 0.01315800 \\ \text { H } & -2.18879000 & 1.03934300 & 1.11756900 \\ \text { H } & 2.95338600 & 0.05249500 & -0.47182300 \\ \text { F } & 1.67523800 & 1.48616300 & 0.13464200 \\ \text { F } & -2.37869100 & 0.52105900 & -0.81314000\end{array}$

Table S28. $\mathrm{X}=\mathrm{Cl}$

$\begin{array}{lrrr}\mathrm{C} & -1.36828000 & 0.61559800 & 0.70865100 \\ \mathrm{C} & 2.15419200 & -0.47900700 & -0.18119900 \\ \mathrm{~S} & 0.58341900 & -1.14612900 & -0.76207500 \\ \mathrm{~S} & -0.57172800 & -0.98960000 & 0.94731900 \\ \mathrm{H} & 2.44301500 & -0.94154300 & 0.75807900 \\ \mathrm{H} & -0.63533200 & 1.37422600 & 0.45337700 \\ \mathrm{H} & -1.84875900 & 0.85080900 & 1.65672500 \\ \mathrm{H} & 2.88606100 & -0.69889000 & -0.95609600 \\ \mathrm{Cl} & 2.17305400 & 1.29599200 & 0.08649000 \\ \mathrm{Cl} & -2.62879000 & 0.63150900 & -0.55947200\end{array}$

\section{Agnostic Conformations of $\mathrm{XH}_{2} \mathrm{C}-\mathrm{S}-\mathrm{S}-\mathrm{CH}_{2} \mathrm{X}$}

Table S29. $\mathrm{X}=\mathrm{Me}$

$\begin{array}{lrrr}\mathrm{C} & -1.79142500 & 0.57525600 & -0.49567800 \\ \mathrm{C} & 1.79142500 & 0.57525800 & 0.49567700 \\ \mathrm{~S} & 0.93070300 & -0.73379000 & -0.44870800 \\ \mathrm{~S} & -0.93070300 & -0.73379000 & 0.44870900 \\ \mathrm{H} & 1.78225000 & 0.29426300 & 1.54863200 \\ \mathrm{H} & -1.24125800 & 1.50966600 & -0.37763900 \\ \mathrm{H} & 1.24125800 & 1.50966700 & 0.37763500 \\ \mathrm{H} & -1.78225200 & 0.29425900 & -1.54863300 \\ \mathrm{C} & -3.21384300 & 0.70813200 & 0.03253800 \\ \mathrm{H} & -3.74732400 & 1.48184300 & -0.52271300 \\ \mathrm{H} & -3.76225500 & -0.22906600 & -0.07909400 \\ \mathrm{H} & -3.21796900 & 0.98360700 & 1.08879600 \\ \mathrm{C} & 3.21384400 & 0.70813100 & -0.03253700 \\ \mathrm{H} & 3.74732400 & 1.48184300 & 0.52271200 \\ \mathrm{H} & 3.76225400 & -0.22906700 & 0.07909700 \\ \mathrm{H} & 3.21797100 & 0.98360500 & -1.08879600\end{array}$


Table S30. $\mathrm{X}=\mathrm{OMe}$

$\begin{array}{lrrr}\mathrm{C} & -1.79600400 & 0.18720600 & -0.33974600 \\ \mathrm{C} & 1.79599600 & 0.18722300 & 0.33973100 \\ \mathrm{~S} & 0.85889100 & -1.09571100 & -0.57275900 \\ \mathrm{~S} & -0.85889200 & -1.09570700 & 0.57276700 \\ \mathrm{H} & 2.02298300 & -0.18754200 & 1.34419700 \\ \mathrm{H} & -1.18489500 & 1.09472000 & -0.41945300 \\ \mathrm{H} & 1.18490200 & 1.09475100 & 0.41938000 \\ \mathrm{H} & -2.02303300 & -0.18759600 & -1.34418900 \\ \mathrm{O} & -2.95541700 & 0.40815400 & 0.40219600 \\ \mathrm{O} & 2.95543900 & 0.40811900 & -0.40218000 \\ \mathrm{C} & -3.84705100 & 1.28356500 & -0.25381900 \\ \mathrm{H} & -4.71166700 & 1.40543000 & 0.39551500 \\ \mathrm{H} & -3.38219500 & 2.26174000 & -0.42597300 \\ \mathrm{H} & -4.16996400 & 0.86716800 & -1.21514500 \\ \mathrm{C} & 3.84704500 & 1.28357700 & 0.25381100 \\ \mathrm{H} & 4.16998800 & 0.86720900 & 1.21513900 \\ \mathrm{H} & 4.71164700 & 1.40547000 & -0.39553600 \\ \mathrm{H} & 3.38215000 & 2.26173500 & 0.42595600\end{array}$

Table S31. $X=F$

$\begin{array}{lrrr}\text { C } & 1.74023500 & 0.52493500 & 0.52840300 \\ \text { C } & -1.74023400 & 0.52493800 & -0.52840200 \\ \text { S } & -0.90633100 & -0.75336200 & 0.49391900 \\ \text { S } & 0.90633300 & -0.75336400 & -0.49391900 \\ \text { H } & -1.93028000 & 0.13290300 & -1.52644300 \\ \text { H } & 1.12829000 & 1.42584900 & 0.56848000 \\ \text { H } & -1.12829100 & 1.42585300 & -0.56847400 \\ \text { H } & 1.93028300 & 0.13289700 & 1.52644200 \\ \text { F } & 2.93365600 & 0.81616200 & -0.07357800 \\ \text { F } & -2.93365900 & 0.81615800 & 0.07357700\end{array}$

Table S32. $\mathrm{X}=\mathrm{Cl}$

$\begin{array}{lrrr}\mathrm{C} & -1.73495600 & -0.37289900 & 0.57318300 \\ \mathrm{C} & 1.73487400 & -0.37309200 & -0.57305500 \\ \mathrm{~S} & 0.92952600 & 0.91037800 & 0.44976000 \\ \mathrm{~S} & -0.92948300 & 0.91034600 & -0.44982700 \\ \mathrm{H} & 1.80861500 & -0.03618600 & -1.60284900 \\ \mathrm{H} & -1.18886000 & -1.31036200 & 0.51181300 \\ \mathrm{H} & 1.18886400 & -1.31059000 & -0.51139900 \\ \mathrm{H} & -1.80889200 & -0.03577300 & 1.60289200 \\ \mathrm{Cl} & -3.37910000 & -0.64597800 & -0.04846800 \\ \mathrm{Cl} & 3.37910500 & -0.64594700 & 0.04845800\end{array}$

